\title{
Newborn Care Practices by the Mother / Care Givers' and Their Knowledge about Signs of Sickness of Neonates
}

\author{
MD. MAHBUBUL HOQUE ${ }^{1}$, MOHAMMAD FAIZUL HAQUE KHAN² ${ }^{2}$, JOTSNAARA BEGUM $^{3}$, MAK AZAD \\ CHOWDHURY ${ }^{4}$, LARS AKE PERSSON 5
}

\begin{abstract}
Background: Despite proven cost effective intervention, there has been little change in neonatal mortality. In Bangladesh neonatal mortality accounts for two third of infants death. About $90 \%$ deliveries take place in home and majority of neonatal death are taking place within 7 days of birth. Information about reasons for delivering at home and newborn care practices will be useful to undertake simple intervention measures by policy makers.
\end{abstract}

Aims and objective: To see the knowledge, perception and behaviour of mothers towards their normal and sick newborn.

Methods: A cross sectional study was carried out in Dhaka Shishu Hospital from June to November, 2007. A semistructured, pretested questionnaire was used to interview mothers attending inpatient (IPD) and outpatient department (OPD) of hospital.

Results: A total 198 mothers were interviewed. Home deliveries were 35.5\% and Institutional were $64.5 \%$. Among the Institutional deliveries $35 \%$ (44 out of 127) were planned and tried first at home, but when failed mothers were taken to hospital. Majority (86\%) of home deliveries were conducted by Dai/relatives. Umbilical cord was cut with new/boiled blade in $85 \%$ of home deliveries and household knife was used in $4 \%$ cases. Birth place were not at all heated in all home deliveries. In $32 \%$ of home deliveries babies were given bath within 1 hour of birth and it was $15 \%$ in case of hospital deliveries. Forty-eight percent babies of home deliveries were wrapped within 10 minutes. Prelacteal feed was given in $51 \%$ of home deliveries in comparison to $23 \%$ of institutional deliveries. The rate of initiation of breast feeding within one hour of birth was $52 \%$ in home and $35 \%$ in institutional deliveries. In all cases breast milk was given within 48 hours. Main reasons cited for delivering at home were preference (43\%) and fear about hospital (39\%). In case of educated (graduate) mothers $72 \%$ deliveries took place at hospital. Less feeding (56\%), vomiting (42\%), less movement (32\%), fever (29\%) and cough (27\%) could be recognized by mothers as signs of sickness.

Conclusion: Home deliveries and poor newborn care practices are commonly found in this study. Traditional birth attendants should be adequately trained as they are conducting majority of home deliveries. Female education is very important to reduce home delivery as it is seen that deliveries of educated mothers are taking place in hospital. High risk traditional newborn care practices like delayed wrapping, early bathing, use of oil in umbilical stump and prelacteal feeding need to be addressed. This study also found that knowledge to identify sickness in newborn is still poor.

1. Associate Professor, Neonatology, Bangladesh Institute of Child Health \& Dhaka Shishu Hospital

2. Medical Officer, Dhaka Shishu Hospital

3. Assistant Professor, Bangladesh Institute of Child Health \& Dhaka Shishu Hospital

4. Professor \& Head, Neonatology, Bangladesh Institute of Child Health \& Dhaka Shishu Hospital

5. Professor, International Maternal \&Child Health, Uppsala University, Sweden

Correspondence: Dr. Md. Mahbubul Hoque

\section{Introduction}

Of the approximately 4 million global neonatal deaths that occur annually, $98 \%$ occur in developing countries, where most neonates dies at home while they are cared by mothers, relatives, and traditional birth attendants. ${ }^{1}$ This high neonatal mortality causes slow declining of infant mortality rate for last 2 decades. Despite proven cost effective intervention to reduce 
neonatal mortality, such as tetanus toxoid immunization, immediate and exclusive breast feeding, and clean cord care, there has been little change in neonatal mortality. ${ }^{2}$ In Bangladesh neonatal mortality accounts for two-third of infant death. ${ }^{3}$ Reducing neonatal mortality will be necessary for achievement the targets for the child mortality under the United Nations MDG.

The World Health Organization's Guidelines for essential newborn care include: hygiene during delivery, keeping the newborn warm, early initiation of breast feeding, exclusive breast feeding, care of eyes, care during illness, immunization and care of low birth weight babies. ${ }^{4}$ But in Bangladesh about $90 \%$ of the deliveries take place at home conducted mainly by untrained birth attendants or relatives in a unhygienic condition. Many of the mothers are very young and uneducated. Majority (about $75 \%$ ) of the neonatal deaths are taking place within 7 days of birth receiving no appropriate care. So initial care and careseeking behaviour at the appropriate place at the appropriate time lies at the hands of the care givers at home. We know the knowledge and care seeking behaviour varies in our population depending on their location, taboos, education, traditional beliefs etc. One needs to know the prevailing situation in the families or the communities to develop ways for improving the situation by imparting appropriate knowledge and undertaking some simple intervention measures. With this view this study was carried out to see the knowledge, perception and behaviour of mothers/ caregivers towards their normal and sick newborn.

\section{Materials and methods}

This cross sectional study was conducted among the mothers and caregivers of the neonates attending Dhaka Shishu Hospital (DSH) from 1st June 2007 to 30th November 2007. DSH is 535 beded pediatric hospital in Bangladesh for primary and tertiary care. Neonatal admission accounts for about $18 \%$ of the total admission in a year. Neonatal mortality contributes about $34 \%$ of the hospital mortality. This hospital receives neonates from different corners of the city as well as from all over the country and comprises mothers from almost all strata of our population. Interviewing these mothers revealed the knowledge and practice regarding newborn care in the various socio-economic strata and geographical location.

Mothers of neonate attending DSH (IPD \& OPD) during this period were interviewed using a structured questionnaire. Comments from open-ended conversation with the mothers were also recorded when relevant. Mothers/caregivers of extremely sick babies and who were unable to give correct information, were not interviewed.

Statistical analysis: The data were subjected to statistical analysis according to standard procedure. SPSS version 12.0 for Windows (SPSS Inc, Chicago, IL, USA) software was used for data recording and analysis.

Ethical issue: Verbal consent was taken from respondents being explained the objectives of the study clearly. Confidentiality and privacy were maintained throughout the study. There was no extrafinancial burden to the patients.

\section{Results}

\section{Socio-demographic characteristics}

Total 198 mothers/ caregivers were interviewed. The mean age of neonates was 12 days (12.14 110.21$)$. The mean age of mothers was 23years (22.66 \pm 4.10$)$. The mean monthly family income was 10,300 taka (approximately 145\$). Most mothers were educated up to primary level (48\%) followed by secondary (23\%). Ten percent mothers were illiterate (Table-I).

Table-I

Distribution of mothers by education

\begin{tabular}{lcccc}
\hline Mother education & $\begin{array}{c}\text { Hospital delivery } \\
\mathrm{N}=83\end{array}$ & $\begin{array}{c}\text { Home+ Failed Home trial. } \\
\mathrm{n}=115\end{array}$ & Total & P vale \\
\hline Primary & $32(33)$ & $63(67)$ & 95 & 0.03 \\
SSC & $23(50)$ & $23(50)$ & 46 & 0.45 \\
HSC & $08(40)$ & $12(60)$ & 20 & 0.63 \\
Graduation & $13(72)$ & $05(28)$ & 18 & 0.01 \\
No education & $07(35)$ & $12(65)$ & 19 & 0.69 \\
\hline
\end{tabular}


Place of delivery, birth attendants:

Home deliveries and Institutional deliveries were $71(35.5 \%)$ and $127(65 \%)$ respectively. Forty four (35\%) cases of institutional deliveries were planned and tried at home first, but when failed mothers were taken to hospital/clinic (Table-II). Ninety-seven deliveries out of $115(71+44)$ were planned for home deliveries, whereas 18 were unplanned (Table-III). Regular antenatal checkup was $10 \%$. Eighty-five percent of home deliveries (61out of 71) were conducted by TBA/Dai and relatives and rest were by nurse (Table-IV).

Table-II

Distribution of mother by place of delivery

\begin{tabular}{lcc}
\hline Place & No & $\%$ \\
\hline Home & 71 & $35.5 \%$ \\
Hospital after failed home trial & 44 & $22.0 \%$ \\
Hospital & 83 & $42.5 \%$ \\
\hline Total & 198 & 100 \\
\hline
\end{tabular}

Cleanliness and hygienic practice:

Umbilical cord was cut with a new boiled blade in $60(85 \%)$ cases of home deliveries and in $3(4 \%)$ cases household knife was used (Table-Va). The stump of umbilical cord was left undressed in $(67 \%)$ cases. But oil (mostly mustard oil) was applied in (14\%) cases and antiseptic solution in $19 \%$ cases (Table-Vc). Most of the neonates were wrapped with old washed cloth $(66 \%)$ and in $46(23 \%)$ cases new washed clothes was used (Table-Vb).

Table-III

Distribution of mothers by reasons for home delivery (included failed home trail): $n-115$

\begin{tabular}{lccc}
\hline Reasons & Planned & \multicolumn{2}{c}{ Unplanned Total(\%) } \\
\hline Prefer home delivery & 18 & 00 & 18 \\
Family members prefer & 25 & 05 & 30 \\
Easy/convenient & 10 & 00 & 10 \\
Previous delivery at home & 10 & 00 & 10 \\
Less cost & 12 & 08 & 20 \\
Fear about hospital & 39 & 00 & 39 \\
\hline
\end{tabular}

Table-IV

Distribution of mother by attendants at home delivery ( $n$-71)

\begin{tabular}{lcc}
\hline Attendants & No & $\%$ \\
\hline Nurses & 10 & 14 \\
TBA/Dai & 56 & 78.9 \\
Relatives & 05 & 7 \\
\hline Total & 71 & 100 \\
\hline
\end{tabular}

Table-V

Cleanliness and hygiene practice

a) Distribution of babies by which babies umbilical cord was cut (home deliveries $n=71$ )

\begin{tabular}{lcc}
\hline Instruments & No & $\%$ \\
New or boiled blade & 60 & 85 \\
Household knife & 03 & 4 \\
Old unboiled blade & 00 & 00 \\
Scissor & 08 & 11 \\
\hline
\end{tabular}

b) Distribution of babies by cloth used for wrapping the baby

\begin{tabular}{lccc}
\hline $\begin{array}{l}\text { Cloth used for } \\
\text { wrapping }\end{array}$ & $\begin{array}{c}\text { Hospital } \\
\text { (including } \\
\text { Failed Home } \\
\text { trial).No (\%) }\end{array}$ & $\begin{array}{c}\text { Home } \\
\text { No (\%) }\end{array}$ & $\begin{array}{c}\text { Total } \\
\text { No (\%) }\end{array}$ \\
\hline Old washed cloth & $89(70)$ & $42(60)$ & $131(66 \%)$ \\
Old unwashed cloth & 00 & $05(7)$ & $05(2.5 \%)$ \\
New unwashed cloth & 00 & $01(1)$ & $01(0.5 \%)$ \\
New washed cloth & $23(18)$ & $23(32)$ & $46(23)$ \\
Unknown & $15(12)$ & 00() & $15(8 \%)$ \\
& $127(100 \%)$ & $71(100 \%)$ & 198 \\
\hline
\end{tabular}

c) Distribution of babies by applicants used in umbilical stump (n-198)

\begin{tabular}{lcc}
\hline Applicants used & No & $\%$ \\
\hline None & 132 & 67 \\
Antiseptic & 38 & 19 \\
Oil & 28 & 14 \\
\hline
\end{tabular}

Maintenance of temperature:

Birth place were not heated in all home deliveries. Bathing of newborn immediately (<1 hour) after birth were practiced in $32 \%$ of home and $15 \%$ of institutional deliveries. In majority cases $56 \%$ of home and $45 \%$ of institutional deliveries were wrapped within 10 minutes and rest $(52 \%)$ were wrapped within 30 minutes (Table-VI). 
Table VI

Practice related to temperature maintenance of baby

a) Distribution of baby by time of bathing:

\begin{tabular}{lcccc}
\hline Time of bathing & $\begin{array}{c}\text { Hospital/(including failed } \\
\text { home trail) } \mathrm{N}=127\end{array}$ & Home $\mathrm{n}=71$ & Total No (\%) & P value \\
\hline Immediately after birth (less 1hr) & $19(15)$ & $23(32)$ & $42(21)$ & 0.007 \\
With in 6 hrs & 00 & $10(2)$ & $10(10)$ & $<0.01$ \\
6-24 hrs, & $10(8)$ & $05(7)$ & $15(7.5)$ & 0.94 \\
more 24 hrs & $40(32)$ & $02(3)$ & $42(21)$ & $<0.01$ \\
more72 hrs & $58(45)$ & $31(56)$ & $89(40.5)$ & 0.97 \\
\hline
\end{tabular}

b) Distribution of babies by time of wrapping at home delivery $n=71$

\begin{tabular}{lcc}
\hline Time(minute) & Home & Percentage \\
\hline$<5 \mathrm{~m}$ & 12 & 17 \\
$5-10 \mathrm{~m}$ & 22 & 31 \\
$10-20 \mathrm{~m}$ & 24 & 34 \\
$20-30 \mathrm{~m}$ & 13 & 18 \\
\hline & 71 & $100 \%$ \\
\hline
\end{tabular}

\section{Feeding}

Breast milk was given as first feed in $59 \%$ cases of institutional and $35 \%$ cases of home deliveries. Infant formula $(18 \%)$ were given as first feed only in institutional deliveries but not in home and cow s milk were given only in home deliveries (14\%) but not in institute. Honey, misri, sugar water were given as prelacteal feed in $51 \%$ of home deliveries in comparison to $23 \%$ of institutional deliveries (TableVII). The rate of initiation of breast feeding within one hour of birth was $52 \%$ in home and $35 \%$ in institutional deliveries. In all cases breast milk was given within 48hours (Table-VIII).

Table-VII

Distribution of babies by type of first feeding

\begin{tabular}{lccc}
\hline Type of first feeding & $\begin{array}{c}\text { Hospital } \\
\text { (including failed } \\
\text { home trail) } \\
\mathrm{N}=127\end{array}$ & $\begin{array}{c}\text { Home } \\
\mathrm{n}=71\end{array}$ & $\begin{array}{c}\mathrm{P} \\
\text { value }\end{array}$ \\
\hline Breast milk & $75(59)$ & $25(35)$ & 0.002 \\
Cow's milk & $00(00)$ & $10(14)$ & $<0.01$ \\
Misri/Sugar/Honey/Water & $29(23)$ & $36(51)$ & $<0.01$ \\
Infant formaula & $23(18)$ & $00(00)$ & $<0.01$ \\
\hline
\end{tabular}

Table-VIII

Distribution of babies by time of initiation of Breast feeding

\begin{tabular}{lcc}
\hline $\begin{array}{l}\text { Timeof initiation } \\
\text { of BF }\end{array}$ & $\begin{array}{c}\text { Hospital (including } \\
\text { failed home trail) } \\
\mathrm{n}=127\end{array}$ & $\begin{array}{c}\text { Home } \\
\mathrm{N}=71\end{array}$ \\
\hline Within 30min & $28(22 \%)$ & $21(31 \%)$ \\
Within 1hrs & $16(13 \%)$ & $15(21 \%)$ \\
Within 2hrs & $55(43 \%)$ & $20(28 \%)$ \\
Within 6hrs & $01(0.7 \%)$ & $05(7 \%)$ \\
Within 24hrs & $17(13.3 \%)$ & $04(6.5 \%)$ \\
Within 48hrs & $10(2 \%)$ & $04(6.5 \%)$ \\
\hline
\end{tabular}

\section{Reasons for home delivery}

Mother herself and or family members' preferred home delivery $(43 \%)$ and fear about hospital $(39 \%)$ were important reasons for planned home deliveries. Precipitate labour was also important reason for unplanned home deliveries (Table-IX).

Mother's Knowledge regarding neonatal sickness: Less feeding (56\%), vomiting (42\%), less movement (32\%), fever (29\%) and cough (27\%) were recognized by mothers as signs of sickness (Table-IX). 
Table-IX

Distribution of mothers/caregivers having knowledge regarding signs\& symptoms of sickness ( $n=198)$

\begin{tabular}{lcc}
\hline Symptoms & Number & Percentage \\
\hline Less feeding & 90 & 45.5 \\
Vomiting & 83 & 42 \\
Less movement & 62 & 31 \\
Hot/ cold & 57 & 29 \\
Excessive crying & 56 & 28 \\
Respiratory distress & 54 & 27 \\
Convulsion & 26 & 13 \\
Bleeding & 26 & 13 \\
Jaundice in <24hrs & 15 & 7.5 \\
Conjunctivitis & 10 & 5 \\
Apnoea & 10 & 5 \\
Cyanosis & 9 & 4.5 \\
No urination after 48hrs & 8 & 4 \\
Oral thrush & 6 & 3 \\
No defecation after 24 hrs & 6 & 3 \\
Delayed cry at birth & 4 & 2 \\
Frothing & 3 & 1.5 \\
Conjunctivitis & 2 & 1 \\
Chocking & 2 & 1 \\
\hline
\end{tabular}

\section{Discussion}

Home deliveries are common in Bangladesh like other developing countries. Our study was in urban area where facilities for institutional delivery are more available than rural area even then home deliveries were $36 \%$ and in another $22 \%$ cases deliveries were tried at home first but when they could not manage mothers were taken to nearby hospital for delivery. Studies done in Nepal and India also showed that home deliveries are common even in urban area. 5,6

Out of 198 cases 114 mothers had no education or only upto primary level. But it was seen that mothers who were highly educated (graduation) there deliveries mostly took place at institution $(P<0.01)$, which is very significant and indicates that education of mothers is very much needed for institutional delivery.

Home deliveries were mostly conducted by traditional birth attendants/Dai (79\%) and in few cases deliveries were conducted by trained attendants. Similar reports were found in a study in Delhi, they found $91.3 \%$ home deliveries were conducted by Dai. ${ }^{7}$ These finding of extremely low presence of skilled birth attendants at home delivery also supported by other studies done in different countries. ${ }^{8,9}$ These suggests that traditional birth attendants should have some basic training regarding delivery process and care of newborn baby as they are attending majority of home deliveries. Training of traditional birth attendants has been shown to be effective in reducing perinatal mortality, showed in a study done in Pakistan. ${ }^{10}$

A new/ boiled blades or scissor were used to cut the cord by $96 \%$ of home deliveries, which is encouraging and similar to other studies . ${ }^{5,6}$ Such a good practice is found to be more common in the present study than earlier studies . ${ }^{11,12}$ We have found that in $4 \%$ cases household knife was used. So mothers and attendants should be made aware of the adverse consequences of using such instrument for cutting the cord.

It is very encouraging that in $65 \%$ cases umbilical stump was kept dry and $17 \%$ cases antiseptic was used. But still in $14 \%$ cases oil was used. This practice of using unsterile substances like oil was similar to the reports from earlier studies done in Nepal and Pakistan. ${ }^{8,13,14}$ This practice of using unsterile substances is a important risk factors for sepsis and tetanus. ${ }^{15}$

Thermal control of newborn is an essential newborn care. We have found that in $32 \%$ of home deliveries and $15 \%$ of institutional deliveries, babies were bathed immediately within one hour of birth. This is much less than previous study done in different countries, 5,6 but it should be discouraged as it can lead to hypothermia. None of the birth place of home delivery were heated during delivery. Only in $17 \%$ of home delivery babies were wrapped immediately ( $<5$ minutes) and rest of the cases it was delayed. This delayed wrapping may cause hypothermia of just born baby.

We have found breast milk as first feed in $52 \%$ cases as compared to $47.5 \%, 32.9 \%, 79.2 \%$ in studies done in Vietnam, Delhi and Nepal respectively. 5,6,16 Use of breast milk as first milk was higher in hospital deliveries( $59 \%$ ) in comparison to home deliveries $(35 \%, \quad P<0.002)$. Practice of prelacteal feed is common in both but more common in home deliveries $(51 \%)$. This difference is significant $(P<0.01)$. Interestingly we found in our study that infant formula was given as first feed only in hospital cases (18\%). 
This is probably a bad effect of illegal visit by the milk companies to the hospitals. Such practices like prelacteal feeding and giving artificial milk are a cause of concern. Such practices delay the initiation of breast feeding and may adversely affect establishment of lactation. ${ }^{17}$

Most mothers in hospital (65\%) and home (50\%) deliveries did not initiate breast feeding within one hour of birth. This is similar to other studies. ${ }^{17,18,19}$ Usefulness of early initiation of breast feeding against the risk of neonatal mortality was established in a study in rural Ghana which showed that $16 \%$ of neonatal death could be avoided if all infants were breast fed from day 1 and $22 \%$ if breast feeding started within $1^{\text {st }}$ hour. ${ }^{20}$ So appropriate counseling to the mothers and care givers regarding early initiation of breast feeding is very important.

In our study reasons for home delivery were related to preferences $(41 \%)$ for home delivery followed by fear about facilities and cost related to delivery. Experiences of previous deliveries at home of family members may be the reason for preference of home delivery and also decision making process in the family about the place of delivery is important. In this study we have also found that educated (graduate) mothers prefer hospital delivery rather than home delivery. So mother's education is very important to reduce home deliveries. Identification of signs of sickness of newborn by mothers or caregiver was poor as found in this study. Less feeding was identified by $56 \%$ of mothers as danger sign. Other signs like vomiting, less movement, fever could be recognized by one third of mothers.

In conclusion, we can say home deliveries and poor newborn care practices are common. Traditional birth attendants should be adequately trained as they are conducting majority of home deliveries. Female education is very important to reduce home delivery as it is seen that deliveries of educated mothers are taking place in hospital. High risk traditional newborn care practices like delayed wrapping, early bathing, use of oil in umbilical stump and prelacteal feeding need to be addressed. Knowledge of mothers to identify sickness in newborn is still poor.

\section{Acknowledgement:}

We acknowledge Swedish International Development Cooperation Agency for their kind support in an Advanced International Training programme of Child
Survival-Reaching the Target. This research was done as a part of the training programme.

\section{References:}

1. World Health Organization. Perinatal mortality: a listing of available information. FRH/MSM.96.7. WHO, Geneva; 1996.

2. United Nations Children's Fund. Trends in childhood mortality in the developing world. New York: UNICEF; 1999, pp. 1960-96.

3. Lawn JE, Cousens S, Zupon J. Neonatal Survival 1: 4 million neonatal deaths: When ? Where? Why? Lancet 2005; 365: 891-900.

4. World Health Organization. Essential newborn care. Report of a technical working group (Trieste, 25-29 April 1994). Geneva: WHO, Division of Reproductive Health (Technical Support); 1996.

5. Sreeramareddy C T, Joshi HS, Sreekumaran B V, Giri S, Chuni N. Home delivery and newborn care practices among urban women in wertern Nepal: a questionnaire survey. BMC Pregnancy and Childbirth 2006; 6: 27-32.

6. Rahi M, Taneja DK, Misra A, Mathur NB, Badhan $S$. Newborn care practices in an urban slum of Delhi. Indian Journal of Medical Sciences 2006; 60(12): 506-13.

7. Bolam A, Munanghar Ds, Shrestha P, Ellis M, Malla K, Costello AM. Factors affecting home delivery in the kathmandu Valley,Nepal. Health Policy Plan 1998;13(2): 152-8.

8. Osrin D, Tumbahangphe KM, Shrestha $D$, et al. Cross sectional, community based study of care of newborn infants in Nepal. BMJ 2002; 325 (7372): 1063.

9. Nepal Demographic and Health Survey. Maternal and Child Health. 2001. p 139-70

10. Jokhio AH, Winter HR, Cheng KK. An intervention involving traditional birth attendants and perinatal and maternal mortality in Pakistan. N Engl J Med 2005;352(20): 2091-9.

11. Hoque A, Selwyn BJ. Birth practices in urban slums of Dhaka, Bangladesh. Women Health 1996; 24(1):41- 58.

12. Nandan $D$, Mishra SK. Delivery practices in west Uttar Pradesh. Indian J Public Health 1996;40(1): 20-1. 
13. Fikree FF, Ali TS, Dhurocher JM, Rahbar MH. Newborn care practices in low socioeconomic settlements of Karachi, Pakistan. Soc Sci Med 2005; 60(5): 911-21

14. Mullany LC, Darmstadt GL, Khatry SK, Tielsch JM. Traditional massage of newborns in Nepal: implication for trial of improved practice. $J$ Trop Pediatr 2005; 51(2): 82-6

15. Bennett J, Azhar N, Rahim F, et al. Further observations on ghee as a risk factor for neonatal tetanus. Int J Epidemiol 1995; 24: 643-7

16. Li L, Thi P L, Hoa NT, Ushijima H. Prevalence of breast feeding and its correlates in $\mathrm{HO}$ Chi Minh City, Vietnam. Pediatr Int 2002; 44(3): 47-54

17. Ahmed FU, Rahman ME, Alam MS. Prelacteal feeding: Influencing factors and relation to establishment of lactation. Bangladesh Med Res Counc Bull 1996; 22(12): 60-4

18. Singh MB, Haldiya KR, Lakshminarayana J. Infant feeding and weaning practice in some semiarid rural areas of Rajasthan. J Indian Med Assoc 1997; 95(11): 576-90

19. Banapurmath $C R$, Nagaraj MC, Banapurmath $S$, Kesaree N. Breast feeding practices in villages of central Karnatka. Indian Pediatr 1996; 336: 477-9

20. Badruddin $\mathrm{SH}$, Inam $\mathrm{SN}$, Ramzanali $\mathrm{S}$, Hendricks K. Constraints to adaptation of appropriate breast feeding practices ina squatter settlement in Karachi, Pakistan. J Pak Med assoc 1997;47:63-8. 